\title{
Null Hypothesis Significance Testing: A Brief Review
}

\author{
Xuejun Ryan Ji, Ph.D. \\ x.ryan.ji@ubc.ca \\ Measurement, Evaluation, and Research Methodology \\ Department of Educational and Counselling Psychology, and Special Education \\ The University of British Columbia, Vancouver, Canada
}

Note: The manuscript was my written prelim exam for my doctorate degree at Texas A\&M University. It was completed in 2015 April, and revised in 2018 August. (C) 2015 XUEJUN RYAN JI ALL RIGHTS RESERVED 


\section{Null Hypothesis Significance Testing: A Brief Review}

Null hypothesis significance testing (NHST) dominates the interpretation of quantitative data analysis in education, psychology, and other social science fields (Shaver, 1993). Meanwhile, the use of NHST has been under enduring and intense criticisms (Carver, 1978; Cohen, 1997; Cumming, 2013; Thompson, 1993, 1996, 1999). In 2015, the journal, Basic and Applied Social Psychology (BASP; Trafimow \& Marks, 2015) banned the use of NHST, reigniting another round of intense discussions about whether continue using the NHST technique.

In the present paper, I have elaborated the definition of NHST and six most common misinterpretations/false beliefs, and suggested reporting strategies, including reporting effect size along with its interval estimates. Finally, I briefly commented on the causes of misconceptions.

\section{Logic and Basic Steps of NHST}

The logic of NHST is essentially an argument by contradiction, designed to show that the null hypothesis will result in a nonsensical conclusion and thus must be rejected (Freedman, Pisani \& Purves, 2007). As the Carver (1978) noted "Statistical significance testing sets up a straw man, the null hypothesis, and tries to knock him down" (p381).

To be more specific, I use a hypothetical example to illustrate the basic concepts of NHST. Suppose that we explore the effects of a tech-based instruction method in a literacy class. In the treatment group, the teacher uses the tech-based instruction method for classroom instruction. In the control group, the teacher uses a typical instruction method. We compare the reading scores of two groups to gauge the treatment effect. This study is well designed and the researcher controls the extraneous variables (e.g., pre-test, school district, SES, teaching experience, etc; in fact, educational research is almost always out of control!). The basic steps of NHST would be: 
1. State null hypothesis (H0) and research hypothesis (H1). H0 is that no difference in reading scores between the two groups after the treatment, or "Tech-based instruction method has no effect on reading ability; or the observed difference is merely due to sampling fluctuations, sampling variation or chance. $\mathrm{H} 1$ is that these two sample groups represent two different populations in terms of reading ability, because different instruction methods were applied.

2. Specify the alpha ( $\left.\mathrm{p}_{\text {cuivas }}\right)$ and determine rejection region $(\mathrm{R})$ by subjective judgment. The alpha is a fixed value and is determined by subjective judgment. It is the probability of rejecting null hypothesis, given the null hypothesis is true.

3. Collect data and calculate the value of test statistics ( $T$, or corresponding $p$-value). The test statistics refer to t, z, F, and chi-square and so forth, based on different test statistic distributions. We can easily obtain test statistics and p-value from statistical software (e.g., SPSS, SAS, STATA etc.).

4. Reject $\mathrm{H} 0$, if the $\mathrm{T}$ value is in the rejection region (or $\mathrm{p}$-value less than $\mathrm{p}_{\text {critias }}$ ); Otherwise, the H0 fails to be rejected. This is a dichotomous reject-or-not-reject decision making procedure. One uses this procedure to decide whether or not to reject the null hypothesis by comparing the $p$-value with the pre-specified $p_{\text {cirial }}$ If $p$-value is less than $p_{\text {civial }}$, then researchers decide to reject the null hypothesis and conclude that a statistically significant result is obtained or the obtained result is not merely due to chance or sample variation. It is noteworthy that we can only use NHST to answer the question of whether an observed difference is a mere chance variation or not. As Carver (1978) noted that:

Statistical significance simply means statistical rareness, Results are "significant" from a statistical point of view because they occur very rarely in random sampling under the 
conditions of null hypothesis (p.383)... Properly interpreted, statistical significance testing provides a p-value or the probability of obtaining mean differences of given sizes under the null hypotheses. Thus, the $\mathrm{p}$ value may be used to make a decision about accepting [better to say NOT rejecting] or rejecting the idea that chance caused the results. This is what statistical significance testing is --- noting more, nothing less. (p.387)

\section{False Interpretations of p-value}

When we make inferences, we must interpret NHST results with caution. We cannot use NHST results to answer the questions that it was not designed for. In the following sections, I discuss six most universal misinterpretations of the p-value in terms of odds-against-chance, the reverse probability error in $\mathrm{H} 0$, validity fallacy, the results replicability, the magnitude of the effect, and the importance of results.

Prior to the discussion, the correct interpretation of the p-value is reviewed. The purpose of NHST is to measure the discrepancy between the sample statistics and the hypothesized values, given the null hypothesis being true (Kline, 2004). Thus, the calculated $\mathrm{p}$ value is a conditional probability of getting the research results, given the null hypothesis is true, say, $\mathrm{p}(\mathrm{D} \mid \mathrm{H} 0)$. Thompson (2006) gave a more detailed definition of the p-value:

The p-value estimates the probability of the sample statistic(s) (and sample results even more extreme in their divergence from the null hypothesis than our sample results), assuming (a) the sample came from a population exactly described by the null hypothesis,and (b) given the sample size.(p.179)

Two assumptions were invoked in the definition: 1) sample size, and 2) population described by the null hypothesis. The sample size affects the estimation precision of sample statistics, and the dispersion of sampling distribution [of statistic]. As the sample size becomes Note: The manuscript was my written prelim exam for my doctorate degree at Texas A\&M University. It was completed in 2015 April, and revised in 2018 August. (C) 2015 XUEJUN RYAN JI ALL RIGHTS RESERVED 
larger, the estimation gets more precise, and so the sample statistics that diverges from the null is less and less likely (Thompson, 2006). In other words, the discrepancy between the sample statistics and what could be in the null is less likely to be explained by chance (or sampling variation), as the estimation precision increases. As for the second assumption, we must assume one single population, so that there is one single p-value for a given set of sample statistics. Otherwise, there might be infinitely many "equally" plausible p-values, if we assume infinitely many populations (Thompson, 2006, p180).

Suppose that in above heuristic example, fortunately, I obtained the p-value of 0.04 , which is statistically significant at the level of 0.05 . First, we have to assume that the null hypothesis is true, the interpretation of $p$ of 0.04 could be:

If the study is repeated many times, $4 \%$ of these results will be extreme or more extreme under the null than the observed result; or $4 \%$ test statistics are further away from the mean of the sampling distribution under the null than the test statistic for the observed result.

To be more specific, in our example, the obtained p-value means 4 in 100 of getting the group mean difference this large or larger than the observed one, given the null hypothesis is true.

However, we CANNOT conclude that:

1. The probability of the chance or sampling error causing the research results is .04 .

2. The probability of the null hypothesis being true is .04 or less than .05 .

3. The chance of the research hypothesis being true is $96 \%$ or more than $95 \%$

4. The likelihood of replication or attainting the statistically significant results is larger than $95 \%$ or say $96 \%$.

5. The $\mathrm{p}$ value of .04 indicates a strong treatment effect.

6. The result is very important. 
False belief 1. The belief that p-value is the probability of chance or sampling error causing the result is called odds-against-chance fantasy (Carver, 1978). When we calculated the p-values, we had assumed that the sampling fluctuations (variations or errors) caused the sample statistic to diverge from the null hypothesis. The probability of sampling error has already been assumed to be 1. Therefore, it is illogical to say that the $\mathrm{p}$ value is the probability of sampling error. In short, we use the $\mathrm{p}$ value to decide whether to reject the null that $100 \%$ chance of sampling errors caused the results.

False belief 2. The belief that the p-value is the probability of the null hypothesis being true. This idea is called an inverse probability error (Carver,1978; Cohen, 1997; Kline,2004). The pvalue is a conditional probability of getting the results, given the null hypothesis is true, $\mathrm{p}(\mathrm{D} \mid \mathrm{H} 0)$, other than the conditional probability of the null hypothesis, given the data or results, $\mathrm{p}(\mathrm{H} 0 \mathrm{ID})$. To tell the difference between these two conditional probabilities, Carver (1978) gave a very concrete example: the probability of obtaining a dead person, given the person was hanged, say, .97 or higher. Reversely speaking, the probability that a person was hanged, given the person is dead might be very low, .01 or lower. There is a clear distinction between these two conditional probabilities. However, this is probably the most universal mistake in interpreting the p-value. The main reason might be that the $\mathrm{p}(\mathrm{H} 0 \mathrm{ID})$ is most researchers want to obtain; however, as Kirk (1996) noted the NHST does NOT tell researchers what they want to know:

Null hypothesis significance testing and scientific inference address different questions. In scientific inference, what we want to know is the probability that the null hypothesis is true given the we have obtained a set of data (D); that is, $\mathrm{p}(\mathrm{HOID})$. What null hypothesis significance testing tells us is the probability of obtaining these data or more extreme data, if the null hypothesis is true, $\mathrm{p}(\mathrm{D} \mid \mathrm{H} 0) .(\mathrm{p} 747)$ 
False belief 3. The belief that the 1-p is the probability of research hypothesis being true, given the data or results, $\mathrm{p}(\mathrm{H} 1 \mathrm{ID})$. This fallacy is called validity fallacy (Mulaik, Raju, \& Narshman, 1997) or valid research hypothesis fantasy (Carver, 1978). As Kline (2004) noted that the complement of p is merely the likelihood of getting the result even less extreme under the null than the observe one, given the null is true. In the heuristic example, the p-value of 0.04 means that 1 in 25 of getting a mean difference this large or larger, and the 1-p, or .96 means 24 in 25 times of getting a mean difference this large or smaller, given the null hypothesis is true. Therefore, 1 - $p$ has nothing to do with $\mathrm{p}(\mathrm{H} 1 \mathrm{ID})$.

False belief 4 . The belief that $1-p$ is the likelihood of replication or attaining the statistically significant results. This fallacy is the replicability fallacy (Carver, 1978; Cohen, 1994). This idea implies that a smaller p-value means that an increasingly greater confidence that the sample results are replicable (Thompson, 1996). Moreover, Nuzzo (2014) echoes the same concerns in her 2014 Nature paper. She revealed that the probability of obtaining another statistically significant result, say, at the level of 0.01 was not $99 \%$, as many falsely assumed, but close to $50 \%$. In fact, as Cumming (2013) noted the p-value is a random variable, demonstrating that the "p-value dances randomly" in a simulation study. Clearly, we cannot use an inherently random measure to evaluate the replicability or stability of research results. The best way to test the replicability is to replicate the study (i.e., external replication; Thompson, 1994). In addition, internal replication analysis can also help researchers obtain the empirical replicability evidence. The methods include the bootstrap, the jackknife and cross-validation. Even though these methods are "never as persuasive as external replication, yet internal methods are far superior to what many researchers use to evaluate result replicability (i.e., "nothing or NHST"; Thompson, 2006, p255).

False Belief 5. The belief that the statistical significance testing directly measures the Note: The manuscript was my written prelim exam for my doctorate degree at Texas A\&M University. It was completed in 2015 April, and revised in 2018 August. (c) 2015 XUEJUN RYAN JI ALL RIGHTS RESERVED 
magnitude of an effect. The p-value is not a useful and reliable measure of results [effect] because it is dependent on sample size (Cohen, 1994; Shaver, 1994). However, $\mathrm{p}$ value and effect size are not completely independent. As Nickerson (2000) pointed out if the sample size and variability are fixed, the larger the effect, the smaller $\mathrm{p}$ is likely to be, and vice versa. However, a very small effect can be also statistically significant, with a very large sample or small variability. Likewise, a large effect can be statistically non-significant, with a very small sample or large variability. Therefore, we cannot use p-value to measure the magnitude of the effect. Instead, we must use effect size to measure the magnitude of the effect, a family of statistics independent of sample size and scale of measurement.

False Belief 6. The belief that statistically significant result is important. Thompson (2006) pointed out “...statistically significant or not statistically significant is the one of most damaging language choice in the history of statistics" (p.147). Even if one concludes that the effect or mean difference is" real" - caused by something other than chance or sampling error, however, it does not indicate that the result is interesting or important. The "significant" in statistics has no relevance to "important" in the value system. As Thompson (1993) explained "if the computer package did not ask you your value prior to its analysis, it could not have considered your value system in calculating p's, and so p's cannot be blithely used to infer the value of research results" (p. 365).

\section{Alternative Statistical Practice}

In the preceding sections, I reviewed the most universal misinterpretations of NHST. Even though NHST has so many controversies, we do not need to ban the use of NHST. As Huberty (1987) noted "there is nothing wrong with statistical tests themselves! When used as guides and indicators, as opposed to a means of arriving at definitive answers, they are okay" (p 7). In short, we cannot ban the use of a particular technique because of its misconceptions and misuse. However, 
due to its limited unity, we must switch our attention from NHST to an alternative statistical practice: reporting effect size and its interval estimates. Such practice is encouraged by the American Psychological Association (APA) Task Force on Statistical Inference. As for effect size and interval estimates, the APA Task Force (Wilkinson \& the APA Task Force on Statistical Inference, 1999, p599) recommended that:

Effect size. Always present effect sizes for primary outcomes. If the units of measurement are meaningful on a practical level (e.g., number of cigarettes smoked per day), then we usually prefer an unstandardized measure (regression coefficient or mean differences) to a standardized measure ( $\mathrm{r}$ or d). It helps to add brief comments that place these effect sizes in practical and theoretical contexts.

Interval estimates. Interval estimates should be given for any effect size involving principal outcomes. Provide intervals for correlations and other coefficients of associations or variations whenever possible. Both effect size and confidence intervals may be misinterpreted as well. Of course, we cannot ban using them. In the following sections, I put more emphasis on the interpretation and practice rather than mathematical calculations.

\section{Effect Size}

Effect size quantifies the extent to which sample statistics diverge from the null hypothesis (Thompson, 2006). There are several types of effect sizes. For example, the two popular effect sizes are Cohen's $d$ and $r^{2}$. Cohen's $d$ is used to measure the group difference, and $r^{2}$ is used to measure the strength of associations. The good reporting practice is to specify which effect size is used in the research rather than simply reporting: "ES =". "ES" is a not "legal" symbol for reporting the effect size. Kirk (1996) gave a nice overview of several measures of the effect magnitude (effect size). In this present paper, we only emphasize the effect size interpretation. 


\section{Interpretation of Effect Size}

Some studies employ Cohen's (1988) benchmarks for "small”, "medium”, and "large effects" to explain the magnitude of the obtained results. As Thompson (2001) pointed out: "if people interpreted effect size with the same rigid that .05 has been used in statistical testing, we would merely be stupid in another metric" ( $\mathrm{p}$ 82-83). The proposed conventions are not eligible to every field, even Cohen (1977) himself caveated that "The qualitative adjective... may not be reasonably descriptive in any specific area.” (p277). Sometimes, a small magnitude effect in one field might be a medium size in another field. The benchmark is not reliable and trustworthy tool to evaluate the effect sizes. Therefore, we must interpret effect sizes within the context and compare them [in terms of magnitude and direction] directly and explicitly in related research (Thompson, 2006). Such practice allows readers to evaluate the stability of effect results across studies (Thompson, 1996). Moreover, reporting effect size can also allow for meta-analysis and power analysis for future studies.

\section{Confidence Intervals}

Confidence Intervals can be calculated for both statistics (e.g., mean) and effect size (e.g., Cohen's d). However, the calculations are different. We can easily obtain the confidence intervals for commonly-used statistics. However, as for confidence intervals for effect size, the computer iteration process must be used (Thompson, 2006). To my knowledge, SPSS and SAS can output effect size (e.g., Cohen's d) by manually inputting formula in the syntax form. Unfortunately, we cannot automatically obtain the confidence intervals for effect size. The newest version of STATA 13, however, can compute both effect size and confidence interval based on either summary statistics or raw data.

Even though the calculations are different, the interpretations (or even misinterpretations) 
are the same. In the present paper, I emphasize the interpretation of confidence interval for statistics. However, the interpretation can be also applied to other confidence intervals.

Interpretation of confidence intervals. The most common misinterpretation of $\mathrm{X} \%$ confidence interval about a sample statistic is that some researchers interpret it as the $\mathrm{X} \%$ of certain (or X\% chance) that the sample statistics lie within the intervals. Such statement is noted as wrong expression in most textbooks. Another similar statement related to $\mathrm{X} \%$ confidence intervals is $\mathrm{X} \%$ confident that the sample statistics lie within interval. This statement is viewed as a correct statement in some textbooks. However, such subtle distinction in wording cannot tell us the 'essence' of the confidence interval.

A single confidence interval, in fact, is one of an infinite sequence of confidence intervals. The certainty level in constructing a given sample confidence interval applies to the construction of infinite confidence intervals drawn from a population, rather than to a single confidence interval in a single sample (Cumming, 2013 ). A 95\% confidence interval means in the long run, 95\% of confidence intervals will include parameter, and 5\% of the interval fail to capture it (Thompson, 2006)

Another misinterpretation of confidence intervals is that some researchers only view them as a double-checking step for the statistical significance test. However, confidence intervals can provide both point estimate and information about the precision of estimate (Thompson, 2006). In other words, confidence intervals provide all of the information from a significance test, and, the range of values within which the true difference is likely to lie (Cumming, 2013).

The good practice to interpret the confidence interval is to compare confidence intervals for parameter estimates across studies (Thompson, 2006). As Schmidt (1996) stated that comparing confidence intervals across related studies helps revealing the true parameters. Such practice can Note: The manuscript was my written prelim exam for my doctorate degree at Texas A\&M University. It was completed in 2015 April, and revised in 2018 August. (C) 2015 XUEJUN RYAN JI ALL RIGHTS RESERVED 
also gauge theoretical progress (Serlin, 1993) and help "prevent[ing] the common mistake of assuming a parameter is contained in a confidence interval" (Wilkinson $\&$ the APA Task Force on Statistical Inference, 1999, p599). Now, one can easily synthesize confidence intervals from different studies by using statistics software packages. For example, Comprehensive Meta-Analysis, can output confidence interval clusters, which allows researchers to make such comparison.

The savior for nonsignificant results in file drawers. Reporting the effect size and its confidence interval might be the "savior" for the nonsignificant results. The nonsignificant test result, for instance, group mean difference, does not mean that there is no difference between two groups; all it means is that the chance error or sampling variance cannot be ruled out as explanation for the observed difference (Kirk, 1996). As for the nonsignificant results, we should not keep them in file drawers, but report the effect sizes and their confidence intervals for future meta-analysis [to minimize the publication bias] (Thompson, 1999).

\section{Conclusion}

Despite the enduring criticisms of NHST, the dichotomous decision-making procedure still dominates the educational and psychology research fields. Most criticisms revolve around the misinterpretation and misuse. However, we cannot ban a technique not because of its own flaws but the fault of the users. The criticisms about NHST has a long history, even since it was invented. Good news is that academic associations such as AERA, APA, and APS have already stepped into the reforms in research practice. Recently, some journal editors already initiated the change. For example, BASP has officially banned NHST. I don't know whether such decision is correct or not, but I believe such step is in the right direction. At least, it draws people's attention to the issues of NHST again. However, the misconceptions of NHST still persists. The misconceptions of p-value might mainly stem from the statistical methodology training. Ironically, in educational research, we Note: The manuscript was my written prelim exam for my doctorate degree at Texas A\&M University. It was completed in 2015 April, and revised in 2018 August. (C) 2015 XUEJUN RYAN JI ALL RIGHTS RESERVED 
always evaluate the effects of the textbook quality and teacher's knowledge on student's academic performance, but we rarely evaluate ourselves.

Note: The manuscript was my written prelim exam for my doctorate degree at Texas A\&M University. It was completed in 2015 April, and revised in 2018 August. (C) 2015 XUEJUN RYAN JI ALL RIGHTS RESERVED 


\section{References}

Carver, R. P. (1978). The case against statistical significance testing. Harvard Educational Review, 48, 378-399.

Cohen, J.(1977). Statistical power analysis for the behavioral sciences. Hillsdale, NJ: Erlbaum.

Cohen, J. (1997). The earth is round (p<. 05) In L.L. Harlow, S. A. Mulaik, \& J. H. Steiger(Eds.), What if there were no significance tests? Psychology Press, IL: Chicago.

Cumming, G. (2013). The new statistics why and how. Psychological Science, 25, 7-29

Freedom, D., Pisani, R., \& Purves (2007). Statistics (4n). New York, NY: W. W. Norton \& Company.

Huberty, C. J. (1993). Historical origins of statistical testing practices: The treatment of Fisher versus Neyman-Pearson views in textbooks. The Journal of Experimental Education, 61, 317-333.

Kirk, R. E. (1996). Practical significance: A concept whose time has come. Educational and Psychological Measurement, 56, 746-759.

Kline, R. B. (2004). Beyond significance testing: reforming data analysis methods in behavioral research. Washington, DC: American Psychological Association.

Mulaik, S., Raju, N. S., \& Narshman, R A. (1997). There is a time and place for significance testing. In L.L. Harlow, S. A. Mulaik, \& J. H. Steiger(Eds.), What if there were no significance tests? Psychology Press, IL: Chicago.

Nickerson, R. S. (2000). Null hypothesis significance testing: a review of an old and continuing controversy. Psychological Methods, 5, 241.

Nuzzo, R. (2014). Statistical errors. Nature, 506, 150-152.

Note: The manuscript was my written prelim exam for my doctorate degree at Texas A\&M University. It was completed in 2015 April, and revised in 2018 August. (C) 2015 XUEJUN RYAN JI ALL RIGHTS RESERVED 
NHST

Schafer, W. D. (1993). Interpreting statistical significance and nonsignificance. The Journal of Experimental Education, 61, 383-387.

Schmidt, F.L. (1996). Statistical significance testing and cumulative knowledge in psychology: Implications for the training of researchers. Psychological Methods, 1, 115-129.

Serlin, R. C. (1993). Confidence intervals and the scientific method: A case for holm on the range. The Journal of Experimental Education, 61, 350-360.

Shaver, J.P. (1993). What statistical significance testing is, and what it is not. The Journal of Experimental Education, 61, 293-316.

Thompson, B. (1993). The use of statistical significance tests in research: Bootstrap and other alternatives. The Journal of Experimental Education, 61, 361-377.

Thompson, B. (1996). AERA editorial policies regarding statistical significance testing: Three suggested reforms. Educational Researcher, 25(2), 26-30.

Thompson, B. (1999). Journal editorial policies regarding statistical significance tests: Heat is to fire as $\mathrm{p}$ is to importance. Educational Psychology Review, 11, 157-169.

Thompson, B. (2001). Significance, effect sizes, stepwise methods, and other issues:

Strong arguments move the field. Journal of Experimental Education, 70, 80-93

Thompson, B. (2006). Foundations of behavioral statistics: An insight-based approach. New York, NY: Guilford

Trafimow, D., \& Marks, M. (2015). Editorial. Basic and Applied Social Psychology, 37, 1-2.

Wilkinson, L., \& APA Task Force on Statistical Inference. (1999). Statistical methods in psychology journals: Guidelines and explanations. American psychologist, 54, 594.

Note: The manuscript was my written prelim exam for my doctorate degree at Texas A\&M University. It was completed in 2015 April, and revised in 2018 August. (C) 2015 XUEJUN RYAN JI ALL RIGHTS RESERVED 